\title{
Fixation et rétention musculaire de la canthaxanthine par la truite arc-en-ciel
}

\author{
G. CHOUBERT et P. LUQUET \\ avec la collaboration technique de R. Cescosse, Y. Hontang et R. Lannebere \\ I.N.R.A., Laboratoire de Nutrition et d'Elevage des Poissons \\ Centre de Recherches Hydrobiologiques \\ Saint-Pée-sur-Nivelle, F 64310 Ascain
}

\section{Résumé}

Un aliment de type commercial contenant $200 \mu \mathrm{g} / \mathrm{g}$ de canthaxanthine de synthèse a été distribué à 3 lots de 100 truites arc-en-ciel (Salmo gairdneri Rich.) d'un poids initial d'environ $125 \mathrm{~g}$ pendant 28 jours. On a suivi, chaque semaine, la quantité de canthaxanthine fixée clans les muscles latéro-dorsaux de 10 poissons pris au hasard.

L'évolution de la quantité de canthaxanthine fixée dans la musculature du poisson (Y) en fonction de la quantité de canthaxanthine ingérée (X) peut être représentée par l'équation hautement significative (seuil 0,01):

$$
\log \mathrm{Y}=-0,35+0,31 \mathrm{X}-0,01 \mathrm{X}^{2}
$$

La quantité de canthaxanthine fixée dans la musculature du poisson tend ainsi à se stabiliser autour d'une valeur de $100 \mu \mathrm{g}$.

L'analyse de régression polynomiale permet de représenter par une équation au second degré hautement significative (seuil 0,01 ) la liaison entre la rétention musculaire $(Y)$ de la canthaxanthine et la quantité de canthaxanthine ingérée (X) par le poisson. Cette équation:

$$
Y=-0,606+0,246 X-0,01 X^{2}
$$

présente un point d'inflexion. La valeur $X_{\max }=12,3 \mathrm{mg}$ correspondant à une rétention maximum $Y_{\max }=0,906$ p. 100 définit la quantité de canthaxanthine ingérée pour laquelle la rétention est maximum. Au-delà, la rétention diminue.

En traçant l'intervalle de confiance $(95 \mathrm{p} .100)$ de la courbe de rétention on peut définir un point $X_{0}$ représentant la quantité de canthaxanthine ingérée pour laquelle la limite supérieure sur la courbe de rétention est égale au maximum de la limite inférieure de la courbe de rétention. Sensu stricto on ne peut dire que la réponse à une quantité ingérée $X$ comprise entre $X_{0}$ et $X_{m i x}$ soit statistiquement différente du maximum de rétention.

\section{Introduction}

La canthaxanthine ( $\beta, \beta$-carotène-4-4'-dione) pigment caroténoïde présent en abondance dans le zooplancton (THOMMEN \& WACKERNagel, 1964 ; KRINSKY, 1965 ; 
Czygan, 1966 ; Davies, Hsu \& Chichester, 1970 ; Czeczuga, 1971, 1973) est un constituant naturel de la nourriture des salmonidés et leur confère une pigmentation rouge (Thommen \& Gloor, 1965). Ce pigment, synthétisé industriellement (IsLER, OFNER \& SiEMERS, 1958) est depuis couramment utilisé tant en aviculture qu'en pisciculture intensive.

Jusqu'alors, la rétention musculaire des pigments caroténoïdes chez les poissons a été étudiée moins sous son aspect dynamique que sous celui des causes de variations. Ainsi, la majorité des auteurs se sont attachés à définir l'influence de la nature de ces pigments et leur concentration dans l'aliment (PETERson et al., 1966; Schmidt \& Baker, 1969 ; Savolainen \& Gyllenberg, 1970 ; Saito \& Regier, 1971 ; Ugletveit, 1974 ; Abdul-Mal.ak et al., 1975 ; Choubert \& Luquet, 1975 ; Kuo et al., 1976; Choubert, 1977, 1979). Quelques études, plus ponctuelles, sont de plus consacrées à l'étude de l'interférence du cycle sexuel (Steven, 1949 ; ShNarevitch \& SaKhnenko, 1971), du facteur génétique (Besse, 1951) ou de l'éclairement des bassins d'élevage (Peterson et al., 1966).

En revanche, l'évolution de la fixation et de la rétention des caroténoïdes en fonction de leur consommation n'a fait, à notre connaissance, l'objet d'aucun travail. Or, ce type d'approche, qui s'est développé chez le poulet (Tagwerker, Streiff \& Brubacher, 1962; BauernfEInd, 1969) apporte des indications précieuses sur l'aptitude des animaux à se pigmenter, sur l'efficacité des différentes matières premières ainsi que sur les concentrations optimales de pigments à incorporer dans les aliments en relation avec la durée de distribution.

Ce sont les raisons pour lesquelles nous avons entrepris l'étude rapportée ci-dessous consistant à suivre, en fonction des quantités consommées, la concentration musculaire en caroténoïdes.

\section{Matériel et méthodes}

L'expérience s'est déroulée dans la pisciculture expérimentale de Donzacq (Landes) qui est alimentée par une eau de source dont la température $\left(17^{\circ} \mathrm{C}\right)$ reste constante toute l'année.

Trois lots de 100 truites arc-en-ciel (Salmo gairdneri Richardson) d'un poids moyen initial de $125 \mathrm{~g}$, sont constitués au hasard et placés dans des bassins expérimentaux rectangulaires $\left(2 \mathrm{~m}^{2} ; 40 \mathrm{~cm}\right.$ de hauteur d'eau).

Pendant 28 jours, ces poissons ont reçu 4 fois par jour, jusqu'à refus, sept jours sur sept, un aliment expérimental renfermant $200 \mu \mathrm{g} / \mathrm{g}$ de canthaxanthine apportée sous sa forme commerciale (produit pur 10 p. 100 hydrosoluble). Cet aliment qui ne renferme pas d'autres pigments caroténoïdes est présenté sous forme de granulés de $5 \mathrm{~mm}$ de diamètre obtenu par pressage sans vapeur (Presse Templewood).

Chaque semaine, un échantillon de 10 truites dans chaque lot est prélevé au hasard pour analyse. Les poissons sont anesthésiés puis tués par rupture des vertèbres cervicales et pesés. Les muscles latéro-dorsaux sont otés et pesés; ces échantillons provenant des différents poissons sont congelés, regroupés et conservés au froid $\left(-18{ }^{\circ} \mathrm{C}\right)$ jusquau broyage qui précède immédiatement les analyses. 
Le dosage de la canthaxanthine est effectué en double sur 30 à $50 \mathrm{~g}$ de tissus frais et $20 \mathrm{~g}$ d'aliment sec, selon la méthode décrite par Osadca, Araujo \& de RITTER (1972). Les résultats obtenus pour les muscles latéro-dorsaux ont été ensuite extrapolés à l'ensemble de la musculature du poisson (LuQueT, 1971 ; Auger, 1973).

La rétention musculaire totale de la canthaxanthine est calculée à partir des variations en concentrations de la canthaxanthine dans les muscles latéro-dorsaux selon la relation:

$$
\text { rétention musculaire }=\frac{(\mathrm{P})(\mathrm{C})_{\mathrm{final}}-(\mathrm{P})(\mathrm{C})_{\text {initial }}}{\text { Consommation en pigment }}
$$

où $\mathrm{P}$ représente le poids des muscles latéraux dorsaux, $\mathrm{C}$ représente la concentration en canthaxanthine des muscles.

\section{Résultats}

\section{1. - Fixation musculaire de la canthaxanthine}

L'évolution de la quantité de canthaxanthine déposée dans la musculature totale du poisson en fonction de la quantité de canthaxanthine ingérée est représentée par une courbe à allure parabolique (fig. 1). La quantité de canthaxanthine fixée dans la musculature du poisson tend ainsi à se stabiliser autour d'une valeur de $100 \mu g$.

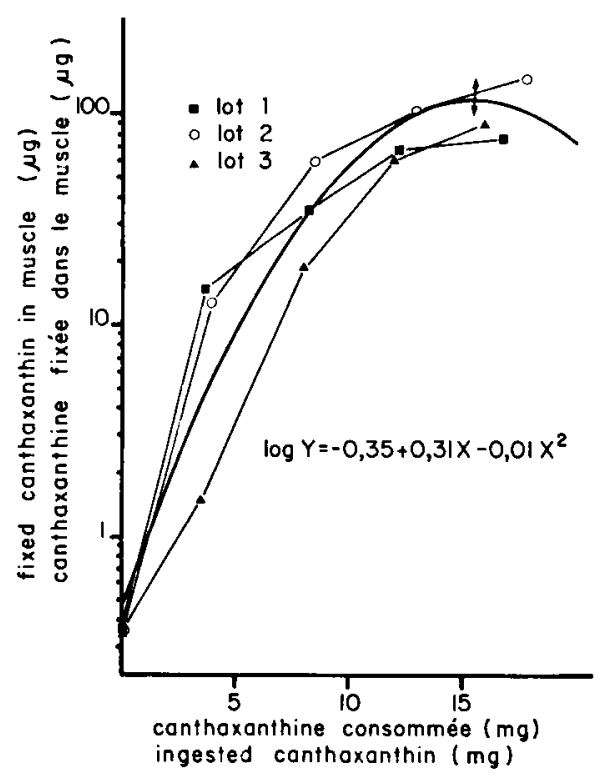

FIG. 1

Corrélation entre la quantité de canthaxanthine ingérée et la quantité fixée dans le muscle des truites.

Correlation between ingested and fixed quantity of canthaxanthin in trout. 
L'analyse de régression polynomiale (SNEDECOR \& Cochran, 1967) permet une estimation de la courbe de régression par une équation du second degré de la forme :

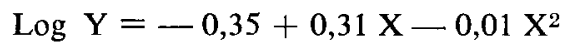

où $Y$ représente la quantité de canthaxanthine fixée dans la totalité de la masse musculaire du poisson, exprimée en $\mathfrak{l g}, \mathrm{X}$ la quantité de canthaxanthine ingérée par le poisson, en $\mathrm{mg}$.

Cette relation est hautement significative $(\mathrm{r}=0,956$ pour $\mathrm{ddl}=11$, $P\{r(11) \geqslant 0,683\}=0,01)$. Cette courbe admet un maximum $Y=120,50 \mu \mathrm{g}$ pour $\mathrm{X}=15,5 \mathrm{mg}$.

\section{2. - Rétention musculaire de la canthaxanthine}

La rétention musculaire de la canthaxanthine cumulée durant les quatre semaines d'expérimentation est rapportée dans le tableau 1 . On remarque une augmentation sensible de la rétention au bout de deux semaines $(\bar{R}=0,458$ p. 100) et trois semaines $(\bar{R}=0,615$ p. 100$)$.

Par l'analyse factorielle (SNEdecor \& Cochran, 1967), la relation entre la rétention musculaire de la canthaxanthine, chaque semaine, et la quantité de canthaxanthine ingérée depuis le début de l'expérience peut s'exprimer par une équation du second degré hautement significative (seuil 0,01 ), de la forme :

$$
\mathrm{Y}=-0,606+0,246 \mathrm{X}-0,010 \mathrm{X}^{2}
$$

où $\mathrm{Y}$ représente la rétention musculaire par semaine en $\mathrm{p} .100, \mathrm{X}$ la quantité de canthaxanthine ingérée en $\mathrm{mg}$.

\section{TABLeaU 1}

Rétention musculaire de la canthaxanthine en fonction du temps d'ingestion du pigment,

\begin{tabular}{|c|c|c|}
\hline $\begin{array}{l}\text { Temps d'ingestion } \\
\text { Ingestion time }\end{array}$ & $\begin{array}{c}\text { Essai (p. 100) } \\
\text { Replicate }(p .100)\end{array}$ & $\begin{array}{l}\text { Moyenne (p. 100) } \\
\text { Mean (p. 100) }\end{array}$ \\
\hline $\begin{array}{l}1 \text { semaine } \ldots \ldots \ldots \ldots \ldots \ldots \\
\text { One week }\end{array}$ & $\begin{array}{l}0,275 \\
0,254 \\
0,034\end{array}$ & $\overline{\mathrm{R}}=0,187 \pm 0,133$ \\
\hline $\begin{array}{l}2 \text { semaines } \ldots \ldots \ldots \ldots \ldots \ldots \\
\text { Two weeks }\end{array}$ & $\begin{array}{l}0,427 \\
0,716 \\
0,232\end{array}$ & $R=0,458 \pm 0,243$ \\
\hline $\begin{array}{l}3 \text { semaines } \\
\text { Three weeks }\end{array}$ & $\begin{array}{l}0,555 \\
0,824 \\
0,505\end{array}$ & $\overline{\mathrm{R}}=0,628 \pm 0,171$ \\
\hline $\begin{array}{l}4 \text { semaines } \\
\text { Four weeks }\end{array}$ & $\begin{array}{l}0,451 \\
0,842 \\
0,554\end{array}$ & $\overline{\mathbf{R}}=0,615 \pm 0,202$ \\
\hline
\end{tabular}
Muscle retention of canthaxanthin relative to the time of ingestion. 
Sur cette courbe de réponse (fig. 2) l'intervalle de confiance (95 p. 100) a été reporté de part et d'autre. La rétention passe donc par une valeur maximum $\mathrm{Y}_{\max }=0,906$ p. 100 quand $\mathrm{X}_{\max }=12,3 \mathrm{mg}$ de canthaxanthine ingérée par le poisson.

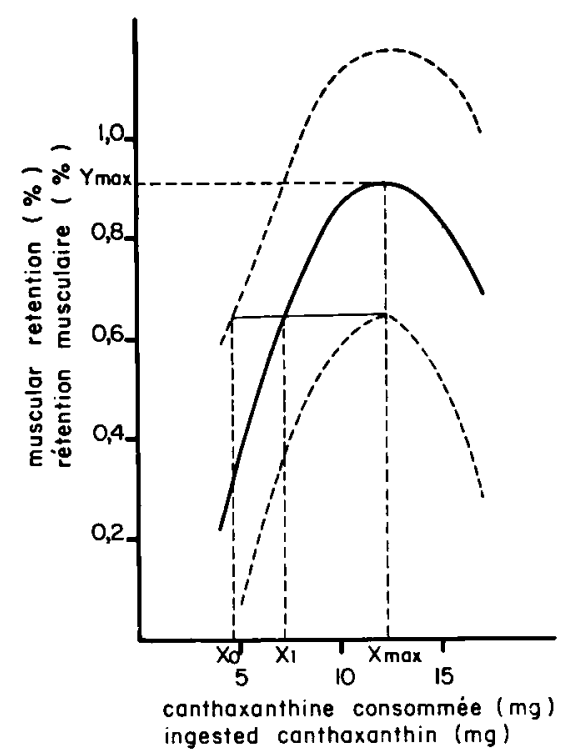

FIG. 2

Rétention musculaire de la canthaxanthine.

Muscle retention of canthaxanthin by trout.

Sur la figure 2 ont été également mentionnés, selon Zeiroun et al. (1976), les points $X_{1}$ et $X_{0} . X_{1}$ représente la quantité de canthaxanthine ingérée correspondant à une rétention égale à la limite inférieure de $\mathrm{Y}_{\mathrm{max}}$, et $\mathrm{X}_{0}$ représente la quantité de canthaxanthine ingérée correspondant à une rétention musculaire égale à la limite inférieure de $Y_{\max }$ sur la courbe représentant la limite supérieure de la rétention musculaire. Les valeurs $X_{0}$ et $X_{1}$ sont respectivement égales à 4,7 et $7,2 \mathrm{mg}$ de canthaxanthine ingérée par poisson.

\section{Discussion}

Le seul examen visuel des muscles de truites ne permet pas de quantifier avec précision la valeur colorante de la canthaxanthine incorporée dans l'aliment des poissons. Pour résoudre de façon plus satisfaisante ce problème, nous avons donc évalué la quantité de canthaxanthine contenue dans les graisses extraites du muscle latéro-dorsal du poisson. 
Nos résuitats montrent que la quantité de canthaxanthine fixée par la truite arc-en-ciel est d'autant plus élevée que la quantité de canthaxanthine ingérée est importante. Toutefois, cette quantité de canthaxanthine musculaire semble atteindre un niveau au-delà duquel toute augmentation de l'ingestion de pigments ne produit plus d'effet. Cette limite se situant aux alentours de 100 g de canthaxanthine fixée par le poisson est encore inexpliquée, mais peut correspondre soit à une impossibilité de la part du poisson à absorber plus de canthaxanthine, soit à une saturation des sites de liaisons lipoprotéiques, comme cela a été noté pour le $\beta$-carotène par Mattews-Roth \& Gulbrandsen (1974). Ces résultats sont à rapprocher, dans une certaine mesure, de ceux obtenus chez la volaille (Tagwerker, StreifF \& BrubaCHER, 1962).

Sur la figure 1, on remarque, également, que le changement de pente de la courbe représentant l'évolution de la canthaxanthine fixée dans la totalité de la masse musculaire du poisson n'a lieu qu'à partir du moment où le poisson a consommé 4 à $5 \mathrm{mg}$ de canthaxanthine. Pour un aliment contenant $200 \mu \mathrm{g} / \mathrm{g}$ de canthaxanthine et un indice de consommation moyen de 1,38 , cela se traduit par une durée de distribution du pigment d'environ une semaine. Chez la poule pondeuse, Marusich, de RitTer \& Bauernfeind (1960) rapportent un phénomène analogue, tant pour la canthaxanthine que pour l'isozéaxanthine et le $\beta$-apo- 8 '-caroténal.

En fait, la quantité totale de canthaxanthine ingérée apparaît plus importante à considérer que le temps pendant lequel les pigments sont consommés. Dans ce contexte, on doit se demander ce qui se passerait si l'administration de la canthaxanthine, en vue d'obtenir une fixation de pigments sur une période de temps plus courte, était effectuée de façon aménagée : une ration dite de démarrage à forte teneur en canthaxanthine précédant une ration dite de finition à teneur moindre en canthaxanthine. Des expériences ultérieures permettront de préciser ces notions.

La rétention musculaire de la canthaxanthine est située à un niveau très bas, inférieur à 1 p. 100 et son évolution est parallèle à celle de la fixation. Par contre, lorsque l'on décompose la rétention musculaire par semaine, on met en évidence l'allure parabolique de son évolution. Cette courbe de rétention présente une valeur maximale au-delà de laquelle les valeurs de rétention diminuent alors que la quantité de pigments ingérés augmente. Cette valeur maximale de 0,906 p. 100 est basse et ne représente environ que le dixième de ce qui est classiquement observé chez la volaille (Mainguy \& Rouques, 1963 ; Ferrando, Mainguy \& Rouques, 1966 ; CheMILLIER, 1977).

Comme le maximum de rétention correspond au point d'inflexion de la courbe de fixation, pour une même quantité de canthaxanthine ingérée, on peut définir qu'une quantité de canthaxanthine ingérée par poisson de $12,3 \mathrm{mg}$ constitue la dose optimale. Ce résultat ne semble pas avoir été rapporté antérieurement.

En portant sur le courbe les points $X_{10}$ et $X_{1}$, précédemment définis, la réponse à une quantité de canthaxanthine ingérée $X$, soit $Y$, comprise entre $X_{0}$ et la quantité de canthaxanthine ingérée correspondant à la valeur maximale de la rétention, n'est pas statistiquement différente de la valeur maximale observée $\left(Y_{\max }\right)$, parce que la distribution $Y_{\max }-Y$ n'est pas connue avec exactitude. C'est pourquoi dans le cas d'un régime alimentaire contenant $200 \mu \mathrm{g} / \mathrm{g}$ de canthaxanthine, analogue à celui employé ici, on peut, pour des raisons économiques évidentes, réduire la quantité de canthaxanthine ingérée par le poisson, c'est-à-dire ramener la durée d'administration. 
de la canthaxanthine de trois semaines (maximum de rétention musculaire) à une période d'environ deux semaines. On peut considérer, en outre, qu'à chaque niveau d'ingestion de la canthaxanthine, la rétention musculaire aurait une allure parabolique dont la valeur maximum serait d'autant plus élevée, et atteinte d'autant plus rapidement que le niveau d'ingestion serait lui-même plus élevé.

Ce faible taux de rétention musculaire de la canthaxanthine est expliqué, tout au moins en partie, par une digestibilité de la canthaxanthine peu élevée, de l'ordre de 5 à 40 p. 100 selon la forme d'apport (CHOUberT \& LuQueT, 1979).

Il importe toutefois de bien souligner qu'il s'agit là de conclusions tirées d'une étude de quatre semaines faite sur de jeunes truites.

\section{Accepté pour publication en février 1982.}

\section{Remerciements}

Nous remercions les Etablissements F. Hoffmann-La Roche et Cie pour la fourniture de Canthaxanthine 10 p. 100 hydrosoluble.

\section{Summary \\ Fixation and retention of canthaxanthin in rainbow trout}

A commercial food containing $200 \mu \mathrm{g} / \mathrm{g}$ of synthetic canthaxanthin was offered for 28 days to 3 groups of 100 rainbow trouts (Salmo gairdneri R.), initially weighing each about $125 \mathrm{~g}$. Every week the quantity of canthaxanthin fixed in the two latero-dorsal muscles was measured in 10 fishes taken at random out from each group.

The relationship (fig. 1) between the quantity of canthaxanthin fixed in the laterodorsal muscles ( $\mathrm{Y}$ ) and the quantity of canthaxanthin ingested by fish (X) can be expressed by the highly significant equation $(0.01)$ :

$$
\log \mathrm{Y}=-0.35+0.31 \mathrm{X}-0.01 \mathrm{X}^{2}
$$

The quantity of fixed canthaxanthin in the fish muscle tend thus to be stabilized around $100 \mu \mathrm{g}$.

A polynomial regression describes best the relationship between canthaxanthin retention ( $Y$ ) in fish and the quantity of canthaxanthin ingested (X). This highly significant $(0.01)$ equation :

$$
\mathrm{Y}=-0.606+0.246 \mathrm{X}-0.01 \mathrm{X}^{2}
$$

shows a point of inflexion (fig. 2). The value $X_{\max }=12.3 \mathrm{mg}$, corresponding to a maximum canthaxanthin retention $=0.906 \mathrm{p}$. 100 , defines the quantity of canthaxanthin ingested corresponding to maximum canthaxanthin retention. Above this level of intake, canthaxanthin retention decreases.

Drawing of the 95 p. 100 confidence limits of this curve allows to determine a point $X_{0}$ representing the quantity of canthaxanthin ingested for which the upper limit on the retention curve equals the maximum of the lower limit on this same curve. Sensu stricto the response to an ingested quantity of canthaxanthin $\mathrm{X}$ comprised between $\mathrm{X}_{0}$ et $\mathrm{X}_{\max }$ cannot be considered as statistically different from the maximum point of canthaxanthin retention.

N.B. Une partie des résultats mentionnés dans cette étude a été présentée au $6^{\mathrm{e}}$ Symposium International sur les Caroténoïdes. Liverpool, Grande-Bretagne, juillet 1981. 


\section{Références bibliographiques}

Abdul-Malak N., Zwigelstein G., Jouanneteau J., Kenig J., 1975. Influence de certains facteurs nutritionnels sur la pigmentation de la truite arc-en-ciel par la canthaxanthine. Ann. Nutr. Aliment., 29 (5), 459-475.

Auger G., 1973. La canthaxanthine, son influence sur la coloration de la chair des truites. Thèse Doc. Vét., Paris, 112 p.

BAUERNFEIND J.C., 1969. Vitamins and carotenoids in modern feeds and animal applications. World Rev. Animal Prod., V, 21-50.

BESSE P., 1951. La saumonisation artificielle des salmonidés, truite et saumon de fontaine. C.R. Acad. Sci., Paris, 233, 637-639.

Chemillier J., 1977. La pigmentation du poulet de chair. Doc. Roche, 1393, 35 p.

Choubert G., 1977. Caroténoïdes fixés par la truite arc-en-ciel en croissance. Thèse Doct. $3^{e}$ cycle, Univ. Paris VI, $47 \mathrm{p}$.

Choubert G., 1979. Tentative utilization of spirulin algae as a source of carotenoid pigments for rainbow trout. Aquaculture, 18 (2), 135-143.

Choubert G., Luquet P., 1975. Nature des caroténoïdes fixés au niveau de la peau et du muscle de la truite arc-en-ciel ayant ingéré de l'huile rouge de Capelan. Ann. Hydrobiol., 6 (2), 123-130.

Choubert G., Luquet P., 1979. Influence de l'agglomération et du stockage des aliments composés sur leur teneur en canthaxanthine. Conséquences sur la digestibilité et la fixation de ce pigment chez la truite arc-en-ciel. Ann. Zootech., 28, 145-157.

Czeczuga B., 1971. Composition and tissue distribution of carotenoids and vitamin $\mathrm{A}$ in the crayfish Astacus leptodactylus. Comp. Biochem. Physiol., 39 B, 945-953.

Czeczuga B., 1973. Carotenoids in some fauna of the Adriatic Sea. III. Leander serratus and Nephrops norvegicus (Crustacea, decapoda). Mar. Biol., 21, 139-143.

Czygan F.C., 1966. Uber den Stoffwechsel von Ketocarotinoiden in niederen Krebsen. Z. Naturforsch., $21 \mathrm{~B}, 801-805$.

Davies B.H., Hsu W.J., Chichester C.O., 1970. Mechanism of the conversion of $\beta$-carotene into canthaxanthin by the brine shrimp Artemia salina. Comp. Biochem. Physiol., 33, 601-615.

Ferrando R., Mainguy P., Rovques A., 1966. Tentatives d'interprétation d'ensemble des effets sur la coloration vitelline de l'apport contrôlé de divers caroténoïdes dans la ration des poules pondeuses. C.R. Acad. Sci., Paris, 263, 676-678.

ISLER O., OfNer A., SIEMERS G.F., 1958. Industrial syntheses of carotenoids for use as food colors. Food Technol., 12, 520-526.

Krinsky N.I., 1965. The carotenoids of the brine shrimp, Artemia salina. Comp. Biochem. Physiol., 16, 181-187.

Kuo H.C., Lee T.C., Kamata T., Simpson K.L., 1976. Red crab processing waste as a carotenoid source for rainbow trout. Alimenta, 2, 2-6.

Luquet P., 1971. Etude du développement chez la truite. Evolution de la teneur en acides nucléiques dans diverses fractions corporelles. Ann. Biol. anim. Bioch. Biophys., 11, 657-668.

Mainguy P., Rouques A., 1963. La couleur vitelline. Doc. Roche, Paris, 63 p.

Marusich W.L., de Ritter E., Bauernfeind J.C., 1960. Evaluation of carotenoid pigments for coloring egg yolks. Poultry sci., 39, 1338-1345.

Mattews-Roth M., Gulbrandsen C.L., 1974. Transport of $\beta$-carotene in serum of individuals with carotenemia. Clin. Chem., 20, 1578-1579.

Osadca M., Araujo M., de Ritter E., 1972. Determination of canthaxanthin in concentrates and feeds. J. Assoc. off. analyt. Chem., 55, 110-113.

Peterson D.H., Jager H.K., Savage G.M., Washburn G.N., Westers H., 1966. Natural coloration of trout using xanthophylls. Trans. Am. Fish. Soc., 95, 408-414. 
Saito A., Regier L.W., 1971. Pigmentation of brook trout (Salvelinus fontinalis) by feeding dried crustacean waste. J. Fish. Res. Bd Can., 28, 509-512.

Savolainen J.E.T., Gyllenberg H.G., 1970. Feeding of rainbow trouts with Rhodoturula sanneii preparation. III. Amounts and qualities of carotenoids. Lebensm. Wiss. $u$. Technol., 3, 18-20.

Schmidt P.J., Baker E.G., 1969. The indirect pigmentation of salmon and trout flesh with canthaxanthin. J. Fish. Res. Bd Can., 26, 357-360.

Shnarevitch I.D., Sakhnenko E.G., 1971. Dynamics of carotenoids in tissues and organs of fish relative to the sexual cycle (en russe). Gidrobiol. Zh., 7 (6), 90-93.

SNedecor G.W., Cochran W.G., 1967. Statistical methods. 6th ed. Iowa State Univ. Press. Ames (U.S.A.), 535 p.

STEVEN D.M., 1949. Studies on animal carotenoids. II. Carotenoids in the reproductive cycle of the brown trout. J. exp. Biol., 26, 295-303.

Tagwerker F.J., Streiff K., Brubacher G., 1962. Carotenes and carotenoids in Poultry nutrition. Proc. 12th World's Poultry Congr., Sydney, 177-181.

Thommen H., Gloor U., 1965. Zum vorkommen von Ketocarotinoiden in der Forelle. Naturwiss., 52, 161-162.

Thommen H., Wackernagel H., 1964. Zur vorkommen von Ketocarotinoiden in Crustacean. Naturwiss., 51, 87-88.

Ugletveit S., 1974. Pigmentering av lakse-og Orretkjett. Fisken Hav. ser. B., 9, 31-60.

Zeitoun I.H., Ullrey D.E., Magee W.T., Gill J.L., Bergen W.G., 1976. Quantifying nutrient requirements of fish. J. Fish. Res. Bd Can., 33, 167-172. 